\title{
Arthrogenic human synovial cysts: immunohistochemical profile of interleukin-1beta, interleukin-6, tumour necrosis factor-alpha
}

\author{
S. Taurone ${ }^{1 *}$, M.T. Santarelli ${ }^{1 *}$, C. De Ponte ${ }^{1}$, L. Bardella ${ }^{2}$, M. Ralli ${ }^{1}$, C. Morselli ${ }^{1}$, A. Nicolai ${ }^{1}$, \\ A. Greco ${ }^{1}$, A. Ferretti ${ }^{3 *}$, M. Artico ${ }^{1 *}$ \\ 'Department of Sensory Organs, "Sapienza" University of Rome, Italy \\ 2Department of Human Neurosciences, "Sapienza" University of Rome, Italy \\ ${ }^{3}$ Orthopaedic Unit and Kirk Kilgour Sports Injury Centre, Sant'Andrea Hospital, "Sapienza" University of Rome, Italy
}

[Received: 28 October 2019; Accepted: 28 November 2019]

Background: Synovial cysts are currently classified as degenerative lesions affecting the joint capsule or adjacent structures.

Materials and methods: In our study we describe the results obtained in an immunohistochemical study comprising 18 patients with synovial cysts, performed to evaluate the pathophysiological role of some inflammatory cytokines such as: interleukin (IL)-1 $\beta$, IL-6 and tumour necrosis factor-alpha (TNF- $\alpha$ ).

Results: Results showed an over-expression of TNF- $\alpha, I L-1 \beta$ and IL-6 which appears to be involved in the onset and progression of the disease. At the present time it is not possible to affirm that these molecules play a direct role also due to the absence of further and more specific investigations. The authors therefore hypothesize that inhibition of inflammation may have a significant role in the pathogenesis and regression of synovial cysts.

Conclusions: Hence, these inflammatory cytokines may be considered potential therapeutic targets. The development of synthetic inhibitors of these inflammatory factors could lead to a reduction in the intensity of inflammation, thus inhibiting the onset and development of the disease. (Folia Morphol 2021; 80, 1: 133-139)

Key words: interleukins 1 beta and 6, immunohistochemistry, synovial cysts, tumour necrosis factor-alpha

\section{INTRODUCTION}

Synovial cysts (also known as "ganglion", "synovial ganglion", "arthrogenic cysts") are benign neo-formations of the soft tissues which generally originate from the joint capsule or tendon sheath and contain fluid gelatinous material, usually appearing on the back of the wrist. Sometimes these cysts may involve neural structures, as in cases of peroneal nerve [1] or lumbar roots [11] involvement.

Arthrogenic cysts are cysts that occur at the level of the joint capsule. The most frequent location is at the wrist. Since the joints are completely wrapped in a fibrous tissue (capsule) that favours the movements' fluidity, a thinner capsular wall is responsible for a possible traumatic damage and so the cyst may develop in the joint. A herniation of the capsule occurs and it tends to move towards the superficial tissues and to form a revolving structure that we call cysts $[4,5]$.

The mechanism that gives rise to these cysts is still not clear. Synovial cysts are mostly found in young athletes or workers who use the wrist and hand joints a lot. In many cases synovial cysts are the result of joint

Address for correspondence: S. Taurone, PhD, Department of Sensory Organs, "Sapienza" University of Rome, V.le del Policlinico 155, 00161 Rome, Italy, tel/fax: 0649918054, e-mail: t.samanta@yahoo.it 
inflammation, arthrosis or previous trauma. However, the exact role of inflammation in this process has not yet been studied in detail. The synovial fluid contains inflammatory factors such as cytokines, prostaglandins and proteases. Previous studies have shown that angiogenic factors are released during the formation of the synovial cysts, suggesting a possible correlation between the proliferation of new vessels in synovial structures and the chronic inflammatory process that induces the progression of synovial cysts $[10,11,13]$. However, to date, no previous studies have analysed the modulation of the inflammatory process in the regression of synovial cysts. It has been shown that in the spinal joint tissue the induction of type 2 cyclooxygenases (COX-2) and phospholipase A2 stimulates the biosynthesis of different inflammatory mediators from synovial chondrocytes (chondrocytes from synovial joints), such as prostaglandin E2, interleukins (IL-1, IL-6, IL-8) and granulocyte-macrophage colony-stimulating factor (GM-CSF) [2]. COX-2 inhibitors have been shown to reduce synovitis, leukocyte infiltration and synovial hyperplasia in animal models, reducing the expression of IL- $1 \beta$, IL- 6 and tumour necrosis factor-alpha (TNF- $\alpha)[7,8]$. Our study is based on previous experimental data that support the role played by inflammatory cytokines and growth factors in the development of arthrogenic synovial cysts. In the light of the data reported in the literature we hypothesize that inhibition of inflammation may play a significant role in the destiny and/or regression of arthrogenic synovial cysts. The aim of the present study was to evaluate the pathophysiological role of some inflammatory cytokines, such as IL-1 $\beta$, IL- 6 and TNF- $\alpha$, in tissue samples of synovial cysts or tenosynovitis, obtained by surgical removal near a wrist joint. The main purpose of this was to evaluate the expression levels and localisation of inflammatory cytokines by immunohistochemical analysis to identify their involvement in the pathology and to evaluate the possible modulation of these factors as a potential therapeutic target.

\section{MATERIALS AND METHODS}

\section{Clinical evaluation}

Written informed consent concerning the donation of human tissues was provided by patients prior to tissue acquisition, following the protocol for the acquisition of human tissues of the Ethical Committee of our University Hospitals which approved the study protocol. All specimens were acquired according to the principles of the Helsinki Declaration.
Eighteen patients were included in the study, aged between 11 and 56 years (11 males and 7 females), undergoing surgical treatment for the removal of wrist synovial cysts, in the Orthopaedic Unit of the S. Andrea Hospital in Rome. Control samples (two specimens for each tissue fragment), characterised by normal palmar fascia tissues, were collected from patients undergoing hand surgery for carpal tunnel syndrome.

Patients with concomitant neoplastic, infectious, autoimmune diseases, peripheral vascular disorders or who had received anti-inflammatory therapy in the 6 months prior to the operation were excluded.

During excision, apart from anaesthesia, no other chemical products or pharmaceutical drugs were administered to the patients. Samples were fixed in formalin and embedded in paraffin to be processed for histological staining and immunohistochemistry. The sections were subjected to haematoxylin and eosin and Masson's trichromic staining.

\section{Immunohistochemistry}

The immunohistochemical analysis was conducted using the $A B C / H R P$ technique (avidin complexed with biotinylated peroxidase) on $4 \mu \mathrm{m}$ thick paraffin sections which were cut using a rotative microtome. These sections were deparaffinized and hydrated through decreasing ethanol series to distilled water, then subjected to microwave irradiation and immersed in citrate buffer $(\mathrm{pH}=6)$ twice for 5 min each time. Subsequently, endogenous peroxidase activity was quenched using $0.3 \%$ hydrogenous peroxide in methanol for $30 \mathrm{~min}$. To evaluate the immunolocalization of IL-1 $\beta$, TNF- $\alpha$ and IL- 6 , the following antibodies were employed: i) rabbit anti-IL-1 $\beta$ polyclonal antibody (1:50, Santa Cruz Biotechnology, Santa Cruz, CA, USA); ii) mouse anti-TNF- $\alpha$ monoclonal antibody (1:100, Santa Cruz Biotechnology, Santa Cruz, CA, USA); iii) rabbit anti-IL-6 polyclonal antibody (1:200; Santa Cruz Biotechnology, Santa Cruz, CA, USA). Incubation with the primary antibodies was performed overnight at $4^{\circ} \mathrm{C}$. Optimal antibody dilution and incubation times were assessed in preliminary experiments. As negative control, the primary antibodies were omitted. After exposure to the primary antibodies all slides were rinsed twice in phosphate buffer $(\mathrm{pH}=7.4)$ and incubated for 1 hour with the appropriate secondary biotinylated antibody at the final dilution of 1:200. The secondary biotinylated antibodies against rabbit and mouse immunoglobulins were 
purchased from Abcam (biotinylated goat anti-mouse antibody and biotinylated goat anti-rabbit antibody). The slides were then incubated with peroxidase-conjugated avidin (Vector Laboratories, Burlingame, CA, USA, Vectastain Elite ABC kit Standard*PK 6-100) for $30 \mathrm{~min}$. Slides were washed in phosphate buffer $(\mathrm{pH}=7.4)$ and treated with $0.05 \%$ 3,3-diaminobenzidine (DAB) and $0.1 \% \mathrm{H}_{2} \mathrm{O}_{2}$. Finally, sections were counterstained with Mayer's haematoxylin and dehydrated rapidly. The staining assessment was made by three experts. The intensity of the immune reaction was assessed microdensitometrically using an IAS 2000 image analyser (Delta Sistemi, Rome, Italy) connected via a TV camera to the microscope. Twelve $100 \mu \mathrm{m}^{2}$ areas were delineated in each section by measuring the diaphragm. The system was calibrated taking the background obtained in sections exposed to non-immune serum as zero.

\section{Statistical analysis}

Statistical analysis was performed with the Student's t-test, using the GraphPad Prism (La Jolla, CA). A p-value $<0.05$ was considered for statistical significance.

\section{RESULTS}

The histopathological alterations caused by human arthrogenic synovial cysts were evaluated using
Table 1. Expression levels of interleukin-1beta (IL-1 $\beta$ ), interleukin-6 (IL-6), tumour necrosis factor-alpha (TNF- $\alpha$ ) in synovial cysts and control specimens, and respective levels of statistical significance (t-test)

\begin{tabular}{lccc}
\hline & $\begin{array}{c}\text { Synovial cysts (\%); } \\
\mathbf{n = 1 5}\end{array}$ & $\begin{array}{c}\text { Control specimens (\%); } \\
\mathbf{n = 1 5}\end{array}$ & P-value \\
\hline IL-1 $\beta$ & $65.27 \pm 12.91$ & $12.61 \pm 5.51$ & $<0.00001$ \\
IL-6 & $60.46 \pm 10.70$ & $11.66 \pm 4.96$ & $<0.00001$ \\
TNF- $\alpha$ & $61.80 \pm 10.22$ & $13.13 \pm 5.81$ & $<0.00001$ \\
\hline
\end{tabular}

The results were considered as statistically significant when $\mathrm{p}$-value $<0.05$.

the immunohistochemical technique. Immunohistochemical experiments have made it possible to visualise the distribution and localization of the cytokines analysed in the tissue samples obtained by surgical removal from patients with arthrogenic synovial cysts (Table 1). Through haematoxylin/eosin staining it was possible to detect, in the pathological tissues, the myo-fibroblastic proliferation site (Fig. A1-A3), not present in the control tissue (Fig. A4). In the pathological tissue (Fig. A1-A3) a thickening of fibroblasts and myo-fibroblasts was visible with areas of complete cellular overlapping. This tissue morphology is not present in normal tissue (Fig. A4), in which only connective tissue with dispersed fibroblasts and small blood vessels can be observed. Figures B1-B3 describe the immunohistochemical expression of
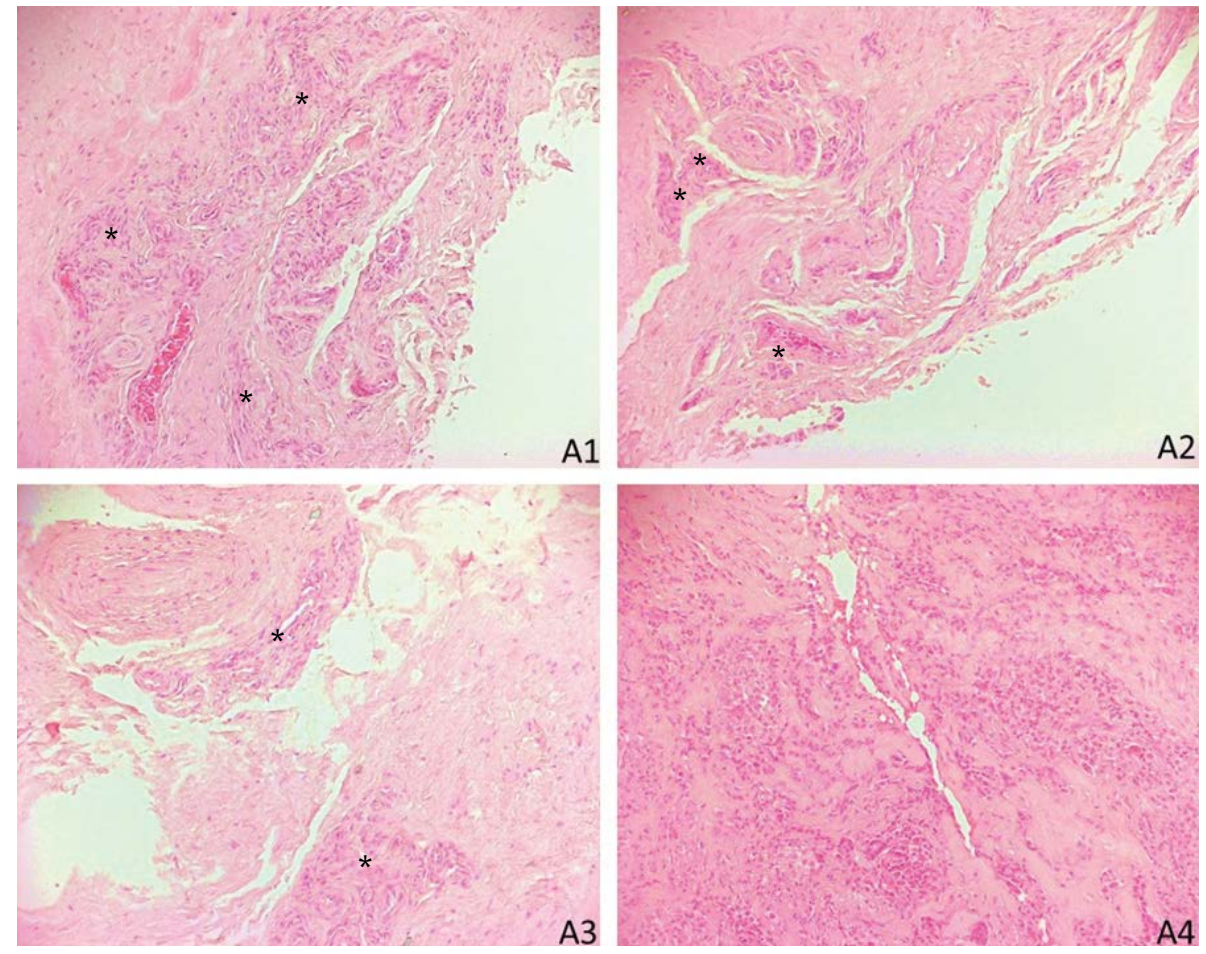

A3
Figure A. Photomicrograph of histological preparations obtained by the haematoxylin/ /eosin staining method; A1-A3. Proliferation myofibroblast nodules (asterisks) obtained by surgical removal from a patient with synovial cyst $(10 \times)$; A4. Normal palmar band consisting exclusively of connective tissue with fibroblasts and blood vessels $(10 \times)$. 

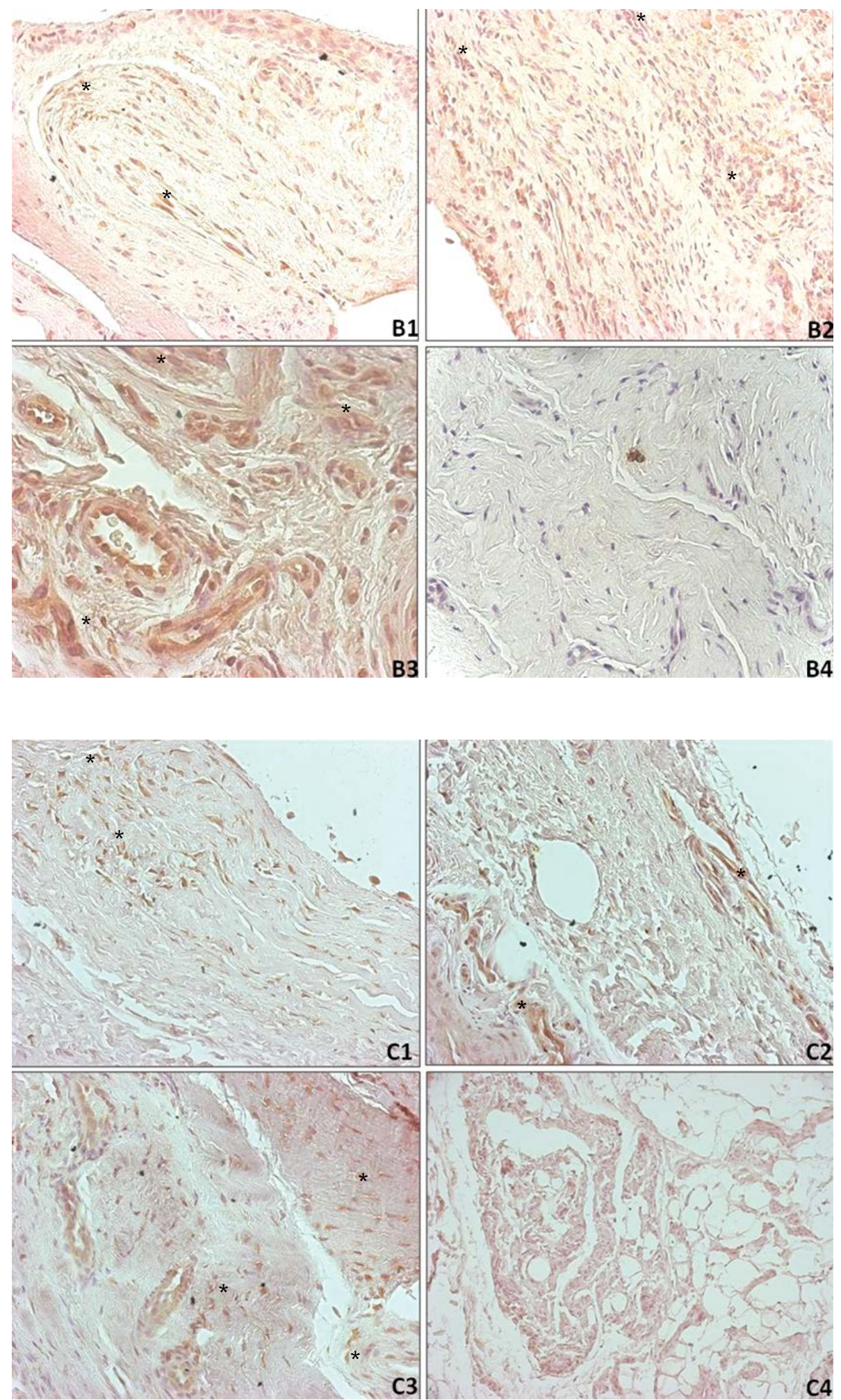

Figure B. Photomicrographs of the immunohistochemical reaction for the inflammatory cytokine interleukin-1 beta (IL-1 $\beta)$; B1-B3. Pathological tissue in which IL-1 $\beta$ is present in the extracellular matrix and in the cytoplasm of fibroblasts and myofibroblasts and in the endothelium of capillaries (B1, B2, 20×; B3, 40×). Myofibroblasts nodules (asterisks); B4. Control tissue $(20 \times)$.
IL-1 $\beta$ in pathological samples, demonstrating positive reactions in the cytoplasm of myo-fibroblastic cells and in the extracellular matrix. IL-1 $\beta$ is completely absent in the extracellular matrix of the palmar control fascia specimens (Fig. B4). Moreover, this pro-inflammatory cytokine is highly expressed at the level of the capillary endothelium, near the synovial cysts. IL-6 is appreciable in extracellular matrix, both in proliferating myo-fibroblasts and in fibroblasts at the level of proliferative nodules in patients affected by arthrogenic synovial cysts (Fig. C1). In the normal tissue of palmar fascia IL- 6 appears to be completely 

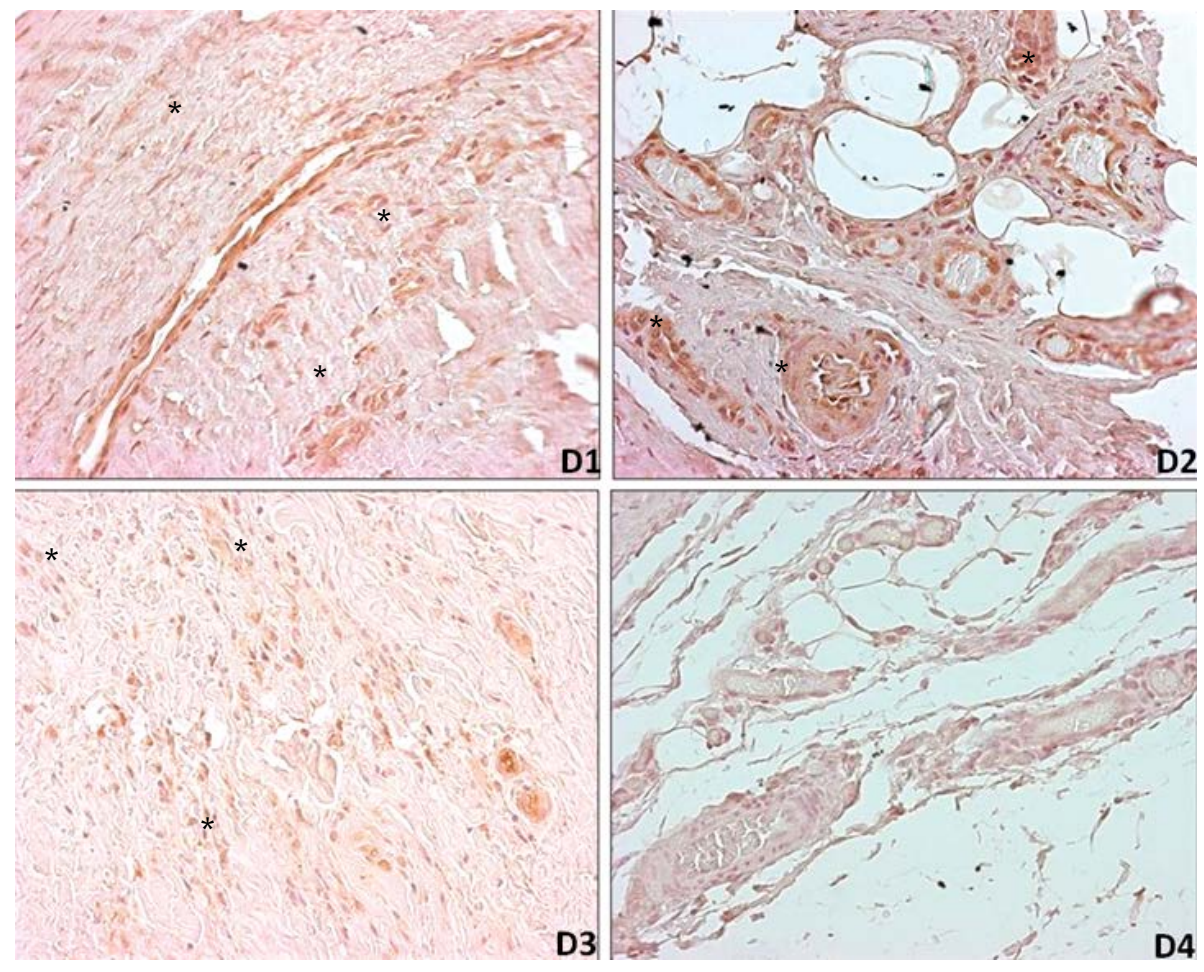

Figure D. Microphotographs of the immunohistochemical reaction for the proinflammatory cytokine tumour necrosis factor-alpha (TNF- $\alpha$ ) expressed in pathological myofibroblasts and in capillary endothelial cells (D1, D2, D3, 20×). Myofibroblasts nodules (asterisks); D4. Control tissue $(20 \times)$.

absent at the level of the loose connective tissue, but moderately present in the endothelial cells of the blood vessels and in the fibroblasts scattered in the connective tissue (Fig. C2). Unlike the other cytokines, IL-6 shows an appreciable localisation in normal fibroblasts (Fig. C1) and a more evident cytoplasmic localisation in the pathological ones (Fig. C2). This cytokine appears to be involved in the inflammatory process that leads to the activation of the fibrotic process. Therefore, IL-6 appears to be synthesized in response to transforming growth factor-beta 1 (TGF- $\beta 1$ ) and acts by enhancing the proliferation and differentiation of fibroblasts into myo-fibroblasts with deposition of amorphous substance in association with TGF- $\beta 1$. TNF- $\alpha$ is expressed in the extracellular matrix of the myo-fibroblastic tissue at the proliferation site observed in patients with synovial cysts (Fig. D1). TNF- $\alpha$ is present in pathological myo-fibroblasts and in capillary endothelial cells (Fig. D1). In the loose connective tissue of the normal palmar fascia this inflammatory factor is however moderately positive in the cytoplasm of fibroblastic cells and in the extracellular matrix (Fig. D2). This growth factor is also strongly expressed in the cytoplasm of secreting sweat glands (Fig. D2) in the dermis near the pathological proliferative nodules.

\section{DISCUSSION}

Arthrogenic synovial cysts are benignly progressing fibro-proliferative disorders which often lead to severe functional damage. The lack of knowledge related to the aetiopathogenesis of the disease has meant that a specific therapy is not currently available until now. Therefore, it is difficult to prevent its onset or to avoid its recurrence after surgical excision. The absence of valid therapeutic targets has led to the development of empirical therapies, such as local injection of steroids [9]. Inflammation plays a fundamental role in the onset of fibrosis and this finding is confirmed by the presence of proinflammatory cytokines [17]. In physiological conditions of wound healing or tissue repair activation of the fibrotic process occurs. During this process fibroblastic cells may differentiate into myo-fibroblasts, because their contractile activity is essential for tissue remodelling. The formation of myo-fibroblasts, controlled by a variety of growth factors and numerous mechanical stimuli, leads to an excessive deposition of extracellular matrix. Their action ends when the tissue is completely repaired or reabsorbed. In some pathological conditions the contractile activity of myo-fibroblasts persists and leads to tissue deformation [16]. IL-1 $\beta$, an important pro-inflammatory 
cytokine, is involved in "in vitro" fibroblastic proliferation through the induction of the expression of transcriptional factors such as c-fos, c-jun and c-myc [6]. The excessive expression of IL-1 $\beta$ alone could be responsible for the local fibroblastic proliferation seen in the active phase of the disease. IL-1 $\beta$ seems to have an important function in the activation and feeding of the inflammatory process, inducing the synthesis of other cytokines such as IL- 6 and IL-2, interferons or chemokines, which are able to attract macrophages and granulocytes towards the site of inflammation. It seems probable that the mitogenic effect of IL-1 $\beta$ is enhanced by the co-expression of other factors such as TGF- $\beta$ and platelet-derived growth factor alpha and beta. The combined expression of these growth factors are probably responsible for fibroblastic proliferation and excessive deposition of an amorphous substance and accumulation of synovial fluid, a condition typical of the disease. In our experiments we also evaluated the level of IL-6 expression. This cytokine is directly involved in the activation of the initial inflammatory process which subsequently leads to fibrosis. The pro-inflammatory cytokine IL-6 plays an important role in the regulation of inflammation and acts in association with TGF- $\beta 1$, thus leading to an increased pro-fibrotic response $[3,14]$. IL- 6 acts by enhancing the TGF- $\beta 1$ signal by increasing endocytosis mediated by non-lipid endosomes. This consequence is due to internalisation of the TGF- $\beta 1$ receptors as a result of binding of their ligand through endocytosis mediated by caveolin lipid vesicles and by non-lipid vesicles, although the TGF- $\beta 1$ signal increases when the receptor endocytosis is mediated by non-lipidic vesicles. Therefore, IL-6 and TGF- $\beta 1$ act synergistically, causing an increase in the expression of proinflammatory cytokines that appear to be the primary cause of the onset of the disease. Verjee et al. [15] reported that TNF- $\alpha$ at low concentrations induce myo-fibroblastic contraction, while at high levels it induces reduction or complete inhibition of myo-fibroblastic contraction. It seems that the action of TNF- $\alpha$ depends strictly on the TNFR receptor type: TNFR2 causes fibroblastic proliferation, while TNFR1 activates programmed cell death. TNF- $\alpha$ could be considered a possible therapeutic target for the treatment of the disease in the primary stages or in preventing relapses following surgical removal. In our experimental study we found that TNF- $\alpha$ showed a greater localisation in pathological fibroblasts. Therefore, this factor appears to be directly involved in the fibrotic reaction and its action depends exclusively on its TNFR2 receptor, which is strongly expressed in pathological conditions. Based upon our preliminary results, local injections of anti-TNF- $\alpha$ drugs could be useful in preventing the progression of the disease or avoiding its recurrence after surgical treatment. During the involutive phase a high ratio of collagen III on collagen I was detected, differently from the normal physiological condition [12].

\section{CONCLUSIONS}

These experimental results suggest, therefore, a possible application of these pro-inflammatory factors in identifying the degree of disease progression and in the use of some of these markers as prognostic factors in the follow-up of patients undergoing surgical resection of synovial cysts. Innovative therapies could be characterised by the combined use of specific inhibitors of the factors TNF- $\alpha$, IL- $1 \beta$ and IL- 6 and their receptors in order to inhibit the progression of the disease through inactivation of the fibrotic process.

\section{Acknowledgments}

This work was supported by a grant of the "Enrico ed Enrica Sovena" Foundation, Italy.

\section{REFERENCES}

1. Artico M, Cervoni L, Carloia S, et al. Synovial cysts: clinical and neuroradiological aspects. Acta Neurochir (Wien). 1997; 139(3): 176-181, doi: 10.1007/BF01844747, indexed in Pubmed: 9143581.

2. Berenbaum $F$, Jacques $C$, Thomas $G$, et al. Synergistic effect of IL-1[beta] and TNF[alpha] on prostaglandin E2 production by articular chondrocytes. Involvement of cyclooxygenase without PLA2 stimulation. Exp Cell Res. 1996; 222(2): 379-384.

3. Bianchi E, Artico M, Di Cristofano C, et al. Growth factors, their receptor expression and markers for proliferation of endothelial and neoplastic cells in human osteosarcoma. Int J Immunopathol Pharmacol. 2013; 26(3): 621-632, doi: 10.1177/039463201302600306, indexed in Pubmed: 24067459.

4. Calderazzi A, Eligi C, Guidetti F, et al. [Synovial chondromatosis of the temporomandibular joint: an occasional finding in association with an arthrogenic cyst. A case report]. Radiol Med. 1995; 89(4): 522-525, indexed in Pubmed: 7597236.

5. De Haas WH, Van Heerde P. Synovial nature of pathologic periarticular structures, including subcutaneous nodules descent from embryonic arthrogenic fibroblasts: a hypothesis. Z Rheumatol. 1979; 38(9-10): 318-329.

6. DiGiovine FS, Duff G. Interleukin 1: the first interleukin. Immunology Today. 1990; 11: 13-20, doi: 10.1016/01675699(90)90005-t.

7. El-Ghazaly MA, Nada AS, El-Hazek RM, et al. Effect of selective COX-2 inhibitor, celecoxib on adjuvant-induced arthri- 
tis model in irradiated rats. Int J Radiat Biol. 2010; 86(12): 1079-1087, doi: 10.3109/09553002.2010.501839, indexed in Pubmed: 20698743.

8. Gebhard HH, Zysk SP, Schmitt-Sody M, et al. The effects of Celecoxib on inflammation and synovial microcirculation in murine antigen-induced arthritis. Clin Exp Rheumatol. 2005; 23(1): 63-70, indexed in Pubmed: 15789889.

9. Huang $A J$, Bos SA, Torriani M, et al. Long-term outcomes of percutaneous lumbar facet synovial cyst rupture. Skeletal Radiol. 2017; 46(1): 75-80, doi: 10.1007/s00256-0162513-5, indexed in Pubmed: 27771754.

10. Kusakabe T, Kasama F, Aizawa T, et al. Facet cyst in the lumbar spine: radiological and histopathological findings and possible pathogenesis. J Neurosurg Spine. 2006; 5(5): 398-403, doi: 10.3171/spi.2006.5.5.398, indexed in Pubmed: 17120888.

11. Nucci F, Artico M, Santoro A, et al. Intraneural synovial cyst of the peroneal nerve: report of two cases and review of the literature. Neurosurgery. 1990; 26(2): 339-344, doi: 10.1097/00006123-199002000-00028, indexed in Pubmed: 2155391.

12. Shih B, Bayat A. Scientific understanding and clinical management of Dupuytren disease. Nat Rev Rheumatol.
2010; 6(12): 715-726, doi: 10.1038/nrrheum.2010.180, indexed in Pubmed: 21060335.

13. Tatter SB, Cosgrove GR. Hemorrhage into a lumbar synovial cyst causing an acute cauda equina syndrome. Case report. J Neurosurg. 1994; 81(3): 449-452, doi: 10.3171/ jns.1994.81.3.0449, indexed in Pubmed: 8057153.

14. Taurone S, Bianchi E, Attanasio G, et al. Immunohistochemical profile of cytokines and growth factors expressed in vestibular schwannoma and in normal vestibular nerve tissue. Mol Med Rep. 2015; 12(1): 737-745, doi: 10.3892/ mmr.2015.3415, indexed in Pubmed: 25738867.

15. Verjee LS, Midwood K, Davidson D, et al. Myofibroblast distribution in Dupuytren's cords: correlation with digital contracture. J Hand Surg Am. 2009; 34(10): 1785-1794, doi: 10.1016/j.jhsa.2009.08.005, indexed in Pubmed: 19910144.

16. Wu M, Ben Amar M. Growth and remodelling for profound circular wounds in skin. Biomech Model Mechanobiol. 2015; 14(2): 357-370, doi: 10.1007/s10237-014-0609-1, indexed in Pubmed: 25183422.

17. Wynn TA, Ramalingam TR. Mechanisms of fibrosis: therapeutic translation for fibrotic disease. Nat Med. 2012; 18(7): 1028-1040, doi: 10.1038/nm.2807, indexed in Pubmed: 22772564. 